\title{
COMPUTATIONAL ACOUSTICS IN SPHERICAL GEOMETRY: STEPS TOWARD VALIDATING HELIOSEISMOLOGY
}

\author{
S. M. Hanasoge, ${ }^{1,2}$ R. M. Larsen, ${ }^{1}$ T. L. Duvall, Jr., ${ }^{3}$ M. L. DeRosa, ${ }^{4}$ N. E. Hurlburt ${ }^{4}$ J. Schou, ${ }^{1}$ \\ M. Roth, ${ }^{5,6}$ J. Christensen-Dalsgahid, ${ }^{7}$ and S. K. Lele ${ }^{2}$ \\ Received 2005 December 12; accepted 2006 May 17
}

\begin{abstract}
Throughout the past decade, detailed helioseismic analyses of observations of solar surface oscillations have led to advances in our knowledge of the structure and dynamics of the solar interior. Such analyses involve the decomposition of time series of the observed surface oscillation pattern into its constituent wave modes, followed by inversion procedures that yield inferences of properties of the solar interior. While this inverse problem has been a major focus in recent years, the corresponding forward problem has received much less attention. We aim to rectify this situation by taking the first steps toward validating and determining the efficacy of the helioseismic measurement procedure. The goal of this effort is to design a means to perform differential studies of various effects such as flows and thermal perturbations on helioseismic observables such as resonant frequencies, travel-time shifts, etc. Here we describe our first efforts to simulate wave propagation within a spherical shell, which extends from 0.2 to about $1.0004 R_{\odot}$ (where $R_{\odot}$ is the radius of the Sun) and which possesses a solar-like stratification. We consider a model containing no flows that will serve as a reference model for later studies. We discuss the computational procedure, some difficulties encountered in a simulation of this kind, and the means to overcome them. We also present techniques used to validate the simulation.
\end{abstract}

Subject headings: hydrodynamics — Sun: helioseismology — Sun: interior — Sun: oscillations — waves

Online material: color figure

\section{INTRODUCTION}

Solar oscillations possess abundant diagnostic information about the solar interior. Helioseismology is the study of the variations in the internal structure and properties of the dynamics of the Sun from measurements of its surface oscillations. Sophisticated observations of these oscillations have led to the inference of the solar structure, the rotation rate, and large-scale dynamics with considerable accuracy. For example, a major result of helioseismology has been the constraint on the solar neutrino flux, which led to a reevaluation of the properties of the neutrino. Helioseismic analyses use the line-of-sight Doppler velocity of plasma at the solar photosphere. This surface is in continual motion due to the interaction, impact, and reflection of millions of wave modes. The primary source of wave generation is the intense turbulence present in the convecting uppermost surface layers. In the Sun, detected waves that possess diagnostic value are either surface gravity or acoustic modes. While surface gravity modes are constrained to sample only the surface layers, acoustic modes plumb the depths of the solar interior and reemerge altered by the structure and dynamics of the solar interior. A substantial part of the wave modes that comprise the acoustic wave

\footnotetext{
${ }^{1}$ W. W. Hansen Experimental Laboratory, Stanford University, 455 Via Palou, Stanford, CA 94305; shravan@stanford.edu.

2 Department of Mechanical Engineering, Stanford University, Stanford, CA 94305 .

${ }^{3}$ Laboratory for Solar and Space Physics, NASA/Goddard Space Flight Center, Greenbelt, MD 20771.

${ }_{5}^{4}$ Lockheed Martin Solar and Astrophysics Laboratory, Palo Alto, CA 94304.

5 Kiepenheuer Institute for Solar Physics, Schöneckstrasse 6, 79104 Freiburg, Germany.

${ }^{6}$ Max-Planck-Institut für Sonnensystemforschung, Max-Planck-Strasse 2, 37191 Katlenburg-Lindau, Germany.

7 Institut for Fysik og Astronomi, Aarhus Universitet, Bygn. 520, Ny Munkegade, 8000 Aarhus C, Denmark.
}

spectrum travel distances large enough that incorporating sphericity into the model becomes unavoidable.

The first validation test involving acoustic simulations of timedistance helioseismology was performed by Jensen et al. (2003), who computed a three-dimensional wave field in a solar-like atmosphere in the presence of a finite-sized sound speed perturbation. The inverted data recovered the main features of the perturbation but was still quite noisy. Little has been done, however, in the context of the forward problem in spherical domains to complement the extensive inversion analyses applied to data obtained from the Michelson Doppler Imager (MDI) on board the Solar and Heliospheric Observatory (SOHO), in operation since 1996.

Our objective is to construct a numerical model that allows waves to propagate within a spherically stratified domain, from which the wave field can be analyzed. The physics of the Sun is governed by an enormous dynamic range, with scales stretching from as short as a meter to as long as several million meters. It is not yet computationally feasible to model this plethora of scales and the phenomena associated with them. Consequently, the goal of this effort is not to produce accurate absolute frequencies to compare with the observations. The aim is to design a careful means to perform differential studies on the effects of largescale flows and asphericities, in the context of global and local helioseismology. The expectation is that the helioseismological signatures of the differences in the acoustic wave field induced by the presence of flows or asphericities are mostly insensitive to the physics we have chosen to discard. Simulations of the wave field in the presence of large-scale perturbations can be compared with a reference case that corresponds to a computation of the wave field in the absence of any flows or perturbations.

In this paper, we discuss computational techniques and the issues that are encountered in a system where thermodynamic properties such as temperature, pressure, and density are strongly spatially varying. We also present techniques of validation that 
were used to demonstrate the verity of this computation. The problem is defined in $\S 2, \S 3$ describes the numerical techniques, $\S 4$ introduces the reader to wave behavior in a solar-like medium, $\S 5$ addresses various computational difficulties, $\S 6$ discusses methods of validation, and in $\S 7$ we summarize and draw conclusions.

\section{PROBLEM DEFINITION}

We solve the three-dimensional linearized Euler equations of fluid motion in a spherical shell encompassing $0.2-1.0004 R_{\odot}$, expanded around the spherically symmetric background state described by model S of the Sun (Christensen-Dalsgaard et al. 1996). The assumption of linearity is justifiable, since acoustic wave velocity amplitudes are much smaller than the background sound speed within the bulk of the computational domain. Because timescales of acoustic propagation are much smaller than the timescale over which large-scale flows or features (of interest to us) change, we assume that the background state is stationary. In the equations that follow, all background quantities are subscripted with a 0 , and all other components are fluctuating.

$$
\begin{gathered}
\frac{\partial \rho}{\partial t}=-\nabla \cdot\left(\rho_{0} \boldsymbol{v}\right)-\nabla \cdot\left(\rho \boldsymbol{v}_{0}\right) \\
\frac{\partial \boldsymbol{v}}{\partial t}=-\nabla\left(\boldsymbol{v}_{0} \cdot \boldsymbol{v}\right)-\boldsymbol{\omega}_{0} \times \boldsymbol{v}-\boldsymbol{v}_{0} \times \boldsymbol{\omega}-\frac{\rho}{\rho_{0}}\left[\nabla\left(\frac{1}{2} v_{0}^{2}\right)+\boldsymbol{\omega}_{0} \times \boldsymbol{v}_{0}\right] \\
-\frac{1}{\rho_{0}} \nabla p-\frac{\rho}{\rho_{0}} g \hat{\boldsymbol{r}}-\Gamma(r) \boldsymbol{v}+S(r, \theta, \phi, t) \hat{\boldsymbol{r}} \\
\frac{\partial p}{\partial t}=-\boldsymbol{v}_{0} \cdot \nabla p-\boldsymbol{v} \cdot \nabla p_{0}-\Gamma_{1} p_{0} \nabla \cdot \boldsymbol{v}-\Gamma_{1} p \nabla \cdot \boldsymbol{v}_{0} \\
\Gamma_{1}=\left(\frac{\partial \ln p}{\partial \ln \rho}\right)_{\mathrm{ad}}
\end{gathered}
$$

Equations (1), (2), and (3) are equations of continuity, momentum, and energy, respectively. The derivative on the righthand side of equation (4) is evaluated along an adiabatic process curve (as denoted by the subscript "ad"). The nomenclature is as follows: $\rho$ is the density, $p$ is the pressure, $\boldsymbol{\omega}$ is the vorticity, $\Gamma_{1}=\Gamma_{1}(r)$ is the first adiabatic exponent, $g$ is gravity, and $\boldsymbol{v}$ is the vector velocity. Equation (3), which states that wave propagation is adiabatic, is justified because the viscous and heat transfer timescales are long in comparison to the acoustic timescales over much of the computational domain.

The term $\Gamma(r) v$ in equation (2) plays the role of a damping agent and $S(r, \theta, \phi, t) \hat{\boldsymbol{r}}$ is the radially directed dipole source. It is believed that wave excitation in the Sun occurs in an extremely narrow spherical envelope (50 km thick) bounded by the surface, and we assume therefore $S(r, \theta, \phi, t)=\tilde{S}(\theta, \phi, t) \delta\left(r-r_{\mathrm{ex}}\right)$, where $r_{\mathrm{ex}}=0.9997 R_{\odot}$ was chosen to be the radial location of the source. The term $\tilde{S}$ is a spatially broadband random function for all but the largest horizontal wavenumbers, which are not included so as to avoid any issues of spatial aliasing. The solar acoustic power spectrum possesses maximum power in the range $2000-5500 \mu \mathrm{Hz}$, with a peak in power around $3200 \mu \mathrm{Hz}$. In order to mimic this excitation behavior, we generate a Gaussian distributed power spectrum with a mean of $3200 \mu \mathrm{Hz}$ and a standard deviation of $1000 \mu \mathrm{Hz}$ in frequency space, which we then Fourier transform to produce a time series with the appropriate source spectrum.
In this study, we perform simulations over a time period that exceeds the time at which the acoustic energy reaches a statistical steady state. The other requirement for the temporal length of the simulation is that the frequency resolution be sufficient for the application at hand. The velocity time series, extracted at the surface, is projected onto a line of sight and used as artificial Doppler velocity data.

\section{NUMERICAL METHOD}

The procedure we employ is pseudospectral; we use a spherical harmonic representation of the spherical surface, sixth-order compact finite differences in the radial direction (see Lele 1992) and a fourth-order, five-stage low dissipation and dispersion rungekutta (LDDRK) time-stepping scheme (see Hu et al. 1996). Latitudes are Gaussian collocation points and longitudes are equidistant. The radial grid is mostly based on the sound speed distribution and is discussed further in $\S 5.1$. The code was developed and run on a multiprocessor SGI-Altix machine at Stanford.

The parallel implementation is in OpenMP with radial locations domain distributed, allowing all the spherical harmonic transforms to be performed in-processor. Spherical harmonic transforms are computed in two steps: longitudinal fast Fourier transforms (FFTs) at each latitude and radius followed by Legendre transforms for each Fourier coefficient and radius. FFTs are performed using the Guru routines provided in FFTW 3.0 and Legendre transforms using matrix multiplication techniques implemented in Level 3 Basic Linear Algebra Subroutines (BLAS). The associated Legendre polynomials $P_{l}^{m}$, where $l$ and $m$ are the spherical harmonic degree and order respectively, are divided into a series of matrices corresponding to different $m$ 's, each of which is further divided into two matrices according to whether $(l-m)$ is even or not. This is done to exploit the symmetry of the associated Legendre polynomials about the equator, which speeds up the transform by a significant amount.

The associated Legendre polynomials are computed according to a highly stable four-term recurrence algorithm given by Belousov (1962), available at the Netlib Web site (the STSWM package). Each transform is a computation of order $O\left(n_{\text {lon }}^{2} n_{\text {lat }} n_{\text {rad }} \log \left(n_{\text {lon }}\right)\right)$, where $n_{\text {lon }}$ is the number of longitudinal gridpoints, $n_{\text {lat }}$ is the number of latitudinal gridpoints, and $n_{\text {rad }}$ is the number of radial gridpoints. To prevent aliasing, we apply the two-thirds rule (Orszag 1970) which sets the lower bound on the number of latitudes at $3 l_{\max } / 2$ where $l_{\max }$ is the maximum $l$ of the simulation. To ensure equal resolution in the latitudinal and longitudinal directions, we set $n_{\text {lon }}=2 n_{\text {lat }}$. Recasting the minimum operation count in term of $l_{\max }$, we obtain an expensive operation count of $O\left(n_{\text {rad }} l_{\text {max }}^{3}\right)$; it is therefore important to minimize the number of times spherical harmonic transforms are performed. Every time step requires the computation of a curl, divergence, and four gradients, each of which involves a computational equivalent of a forward-inverse transform pair. Level 3 BLAS is known to operate near the peak performance of the processor, so these computations are generally very efficient, when they are performed in-processor.

We place transmitting boundary conditions (Thompson 1990) at both radial boundaries of the computational domain. While this particular boundary condition is most effective at absorbing waves that are of normal incidence, it reflects a significant percentage of all other waves. To mitigate this effect, we introduce an absorbent buffer zone (see, e.g., Lui 2003; Colonius \& Lele 2004), placed in the evanescent region, that damps waves out substantially before they reach the boundary. This is one of the purposes that the term $\Gamma(r)$ in equation (2) fulfills. 


\section{ACOUSTIC WAVE PROPAGATION IN THE SUN}

Acoustic modes are uniquely identified by three parameters, the spherical harmonic degree $l$, azimuthal order $m$, and radial order $n$. A detailed description of this classification system and more on wave behavior as a function of these parameters can be found in chapter 5 of Christensen-Dalsgaard (2003). Because sound speed increases with depth, waves that are initially propagating at a nonzero angle to the upper boundary refract continually as they propagate deeper, until they undergo total internal refraction at some depth and reemerge at the surface. At the point of total internal refraction, also known as the inner turning point, the wave is propagating purely horizontally. To determine the location of the inner turning point we proceed as follows. The dispersion relation is

$$
\omega=c k,
$$

where $\omega$ is the frequency, $c$ the sound speed, and $k=|\boldsymbol{k}|$, where $\boldsymbol{k}$ is the vector wavenumber. We decompose $\boldsymbol{k}$ into a radial $\left(k_{r} \hat{\boldsymbol{r}}\right)$ and a horizontal $\left(\boldsymbol{k}_{h}\right)$ component according to

$$
\boldsymbol{k}=k_{r} \hat{\boldsymbol{r}}+\boldsymbol{k}_{h}
$$

We have, using equations (5) and (6),

$$
k_{r}^{2}+k_{h}^{2}=\frac{\omega^{2}}{c^{2}},
$$

where $k_{h}=\left|\boldsymbol{k}_{h}\right|$, the horizontal wavenumber, is given by

$$
k_{h}(r)=\frac{\sqrt{l(l+1)}}{r} .
$$

To determine the inner turning point, $r_{t}$, we set $k_{r}=0$, and obtain

$$
\frac{c\left(r_{t}\right)}{r_{t}}=\frac{\omega}{\sqrt{l(l+1)}} .
$$

This relation thus provides us a means to identify the maximum penetration depth of each wavemode. The inverse is true as well: the frequency-wavenumber range of waves that can access a given depth may be determined from equation (9).

\subsection{Upper Turning Point: Reflection at the Surface}

The acoustic cutoff frequency $\omega_{A}$, below which waves become evanescent, is related to the density scale height, $H_{\rho}$, in the following manner (Deubner \& Gough 1984)

$$
\omega_{A}=\frac{c}{2 H_{\rho}}\left(1-2 \frac{d H_{\rho}}{d r}\right)^{1 / 2} .
$$

We define $H_{\rho}$ as

$$
H_{\rho}=-\left(\frac{d \ln \rho}{d r}\right)^{-1}
$$

and in a similar manner, the pressure scale height, $H_{p}$, as

$$
H_{p}=-\left(\frac{d \ln p}{d r}\right)^{-1} .
$$
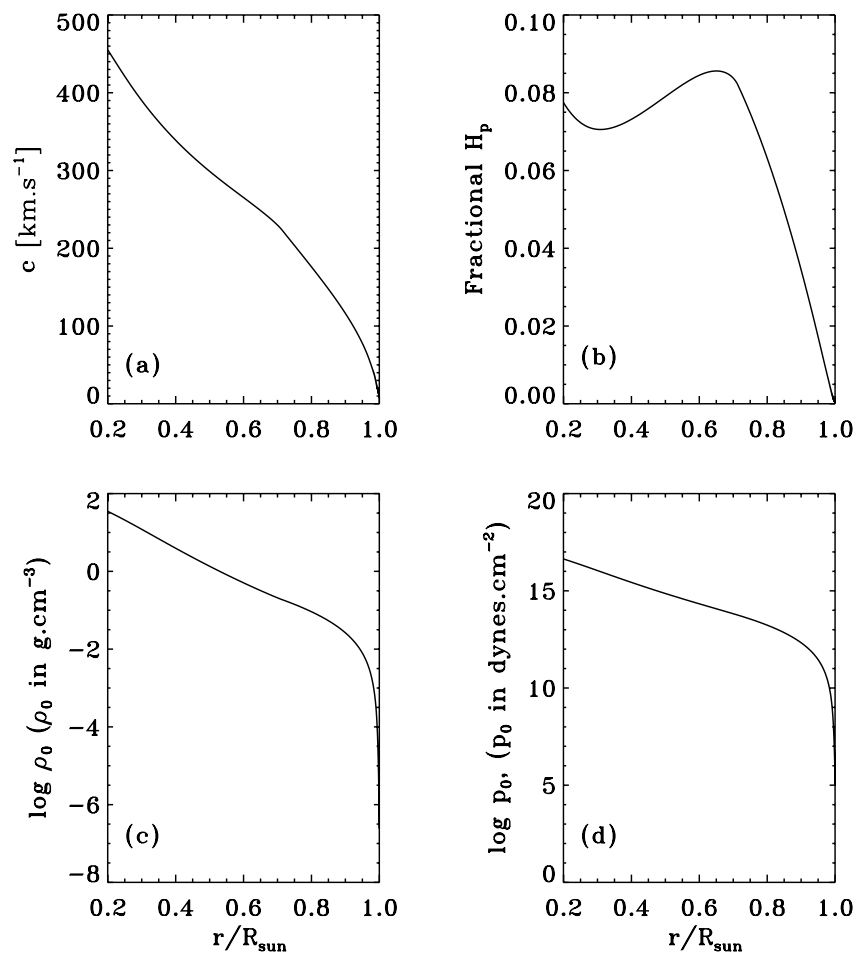

FIG. 1.-Background properties from model S of the Sun (ChristensenDalsgaard et al. 1996) as functions of radius. The horizontal coordinate of all the panels above is the fractional radius, $r / R_{\odot}$. Panel $(a)$ shows the dependence of sound speed (in $\mathrm{km} \mathrm{s}^{-1}$ ) with $r / R_{\odot}$. Panel $(b)$ contains the fractional pressure scale height variation $\left(H_{p} / R_{\odot} ; H_{p}\right.$ has been defined in eq. [12]) with the fractional radius. Note the rapidly decreasing scale height in the near-surface layers. Panels $(c)$ and $(d)$ show logarithmic variations in density and pressure as a function of the fractional radius.

As waves propagate toward the surface, the density and pressure scale heights become increasingly small, as depicted in Figure 1. The dependence of the cutoff frequency as a function of radius is shown in Figure 2. Waves possessing frequencies higher than $6000 \mu \mathrm{Hz}$ or so escape into the atmospheric layers, and they are of little interest to us. The rest of the spectrum is reflected at locations, also known as the upper turning points, where the wave frequency equals the cutoff frequency. This property was taken into account when choosing the excitation parameters. A useful consequence of this arrangement is that above their upper turning points, waves with frequencies less than the cutoff become evanescent. This aids the design of a transparent boundary condition, since these waves can be damped into nonexistence much more easily.

\section{COMPUTATIONAL ISSUES}

Computationally speaking, the properties of the Sun are relatively well behaved and comparatively easy to model up to about $r=0.98 R_{\odot}$. The near-surface layers however, introduce the multiple difficulties of rapidly dropping density height scales, increasingly unstable stratification, the presence of an ionization zone, complexity in the equation of state, and nonlinearities into the wave propagation physics. Added to these issues is the fact that acoustic waves spend most of their time in the near-surface layers because the sound speed is smallest there. The consequences of not taking into account some or all of the complex near-surface dynamics is not entirely clear because the issues listed above are inextricably linked to each other.

Keeping in mind the important issue of computational feasibility, we cannot hope to resolve the complex small-scale physics 


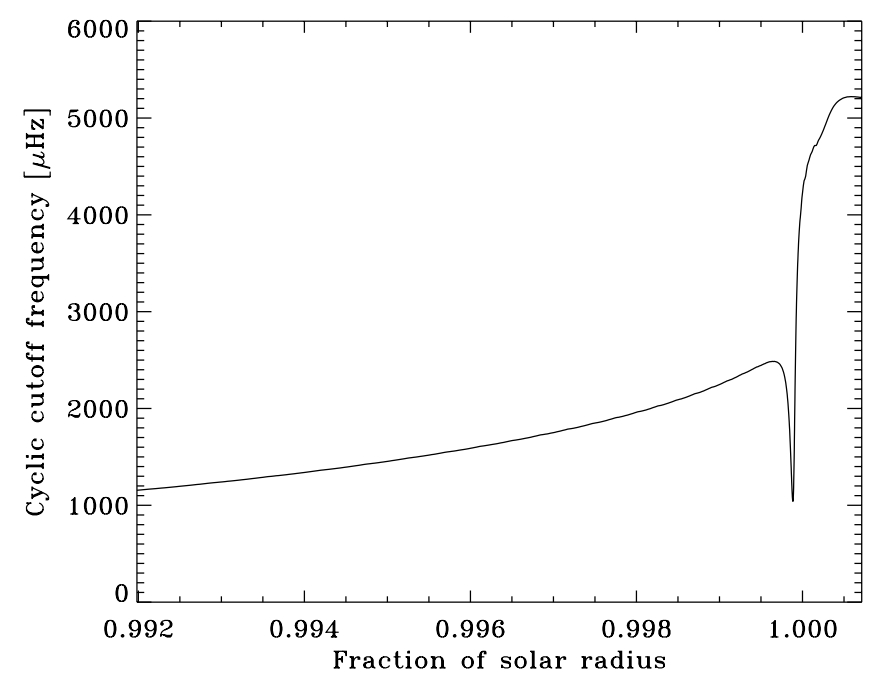

FIG. 2.-Acoustic cutoff frequency $\omega_{A} / 2 \pi$ as a function of radius in the model. The steep rise in $\omega_{A}$ in the near-surface layers is due to rapid changes in the density scale height, and causes outward-propagating waves with frequencies $\omega<\omega_{A}$ to reflect and propagate inward.

of the near-surface layers or model the convecting solar interior. In fact, we see the formalism presented in this article as complementary to the work of, e.g., Rosenthal et al. (1999) and Georgobiani et al. (2003), who perform detailed hydrodynamical simulations of the near-surface layers to extract information about their effects on the frequencies and excitation of the modes. We reiterate that the method presented here is a means to study the differential effects of flows and asphericities on various helioseismological measures, such as resonant frequencies, travel-time anomalies, etc.

\subsection{Choice of Radial Grid}

To determine an appropriate radial grid, we have taken into account the strong radial dependence of background solar properties such as pressure, density, and sound speed. Consider a wave propagating at the speed of sound in the radial direction according to the simple advection equation

$$
\frac{\partial u}{\partial t}+c(r) \frac{\partial u}{\partial r}=0 .
$$

It makes immediate sense to choose a grid stretching function

$$
\tau(r)=\int_{r} \frac{d x}{c(x)},
$$

that transforms equation (13) to

$$
\frac{\partial u}{\partial t}+\frac{\partial u}{\partial \tau}=0
$$

a form that is much easier to handle. The relation between two adjacent grid points then is

$$
\begin{gathered}
\int_{r_{i}}^{r_{i+1}} \frac{d r}{c}=\delta, \\
\delta=\frac{1}{n_{\mathrm{rad}}-1} \int_{r_{\text {in }}}^{r_{\mathrm{out}}} \frac{d r}{c},
\end{gathered}
$$

where $r_{\text {in }}, r_{\text {out }}$ are the inner and outer radii respectively and $n_{\text {rad }}$ is the number of radial grid points including the boundaries.

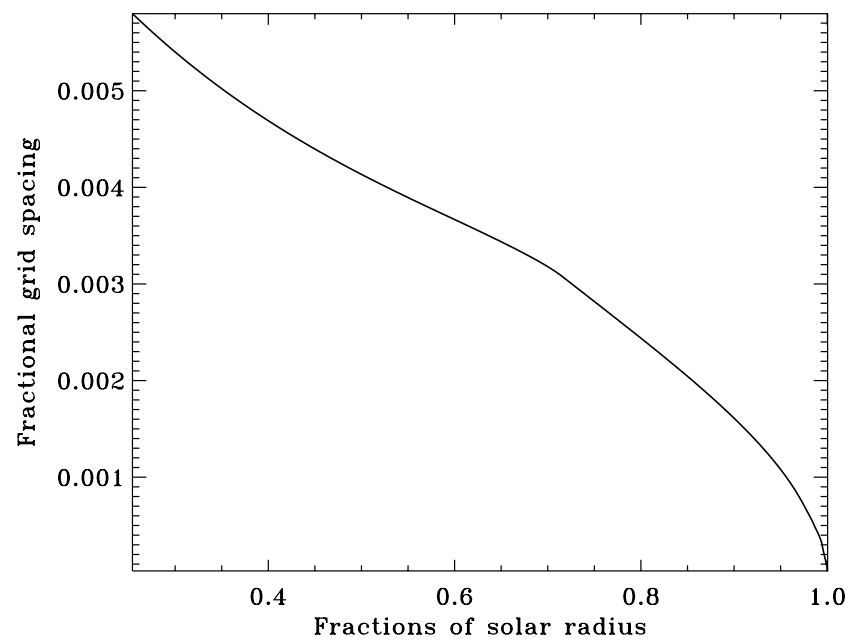

FIG. 3.-Fractional grid spacing as a function of radius for $n_{\text {rad }}=350$. Plotted is $d r / R_{\odot}$, where $d r$ is the local grid spacing, as a function of the fractional radius, $r / R_{\odot}$. For $r \leq 0.99 R_{\odot}$, the grid spacing is chosen to maintain the constancy of the traveltime of an acoustic wave between adjacent grid points. To account for rapidly decreasing scale heights, the radial grid points from $0.9915 R_{\odot}$ to the upper boundary are equally spaced in $\ln p$. Third order splines are used to vary the grid spacing between 0.99 and $0.9915 R_{\odot}$ as smoothly as possible.

Since sound speed is a monotonically decreasing function of radius, the radial grid spacing becomes larger at depth. It is also important to note that gradients of background quantities become smaller with depth, so it makes sense that the grid is coarser.

However, as noted in $\S 4$, pressure and density scale heights tend to become very small at the surface, and pressure and density fall much more rapidly with radius than sound speed. It is therefore useful to choose a different heuristic for the grid spacing for the outer layers, perhaps along the lines of a logarithmic pressure grid. In this simulation, we have adopted a constant travel-time grid for $r \leq 0.99 R_{\odot}$, patched smoothly with one equally spaced in $\ln p$ from $0.9915 R_{\odot}$ to the upper boundary. Third-order splines are used to vary the grid spacing between 0.99 and $0.9915 R_{\odot}$ as smoothly as possible. Figure 3 displays the grid spacing as a function of radius.

\subsection{CFL Restrictions}

The Courant-Friedrich-Lewy (CFL) condition determines the maximum size of the time step based on spatial resolution and, in this case, sound speed. The time step is limited by the more restrictive of the accuracy and stability conditions (see Hu et al. 1996). The accuracy condition requires that well-resolved waves are captured to within accepted numerical error, and the stability condition ensures that the highest resolved wavenumber is stable. Since waves travel at various angles, we must consider limitations due to the horizontal wavenumber as well.

The shortest wavelength that can be accommodated on the radial grid (without any aliasing) is twice the largest grid spacing, which is the distance between the deepest two grid points. Since the spatial differencing scheme can only capture derivatives with wavenumbers in a given range, we introduce two relevant measures (e.g., Lele 1992; Lui 2003) used to describe this effect: the highest well-resolved wavenumber and the highest resolved wavenumber. The highest well-resolved wavenumber represents the largest wavenumber that is resolved accurately by the differencing scheme. The highest resolved wavenumber is the maximum effective wavenumber of the spatial differencing scheme. The highest well-resolved radial wavenumber, which we refer to as $k_{w}$, and highest resolved radial wavenumber, $k_{w n}$ 
for the choice of our differencing scheme are $k_{w} h=1.7$ and $k_{w n} h=2.0$, where $h$ is the local radial grid spacing. The highest well-resolved horizontal wavenumber (for a spectral method, there is no difference between the highest resolved and highest well-resolved wavenumbers) is given by

$$
k_{h, \max }=\frac{\sqrt{l_{\max }\left(l_{\max }+1\right)}}{r_{\text {in }}},
$$

where $l_{\max }$ is the spherical harmonic bandwidth. The time step restriction is given by

$$
\Delta t \leq \min \left[\frac{L}{\left(c k_{w}\right)_{\max }}, \frac{R}{\left(c k_{w n}\right)_{\max }}, \frac{L}{\left(c k_{h}\right)_{\max }}\right],
$$

where $R$ and $L$ are limited by the boundary of the stability footprint and the accuracy limit, respectively (Hu et al. 1996). The time-stepping scheme adopted in this calculation is accurate and stable for $L \leq 1.35$ and $R \leq 3.54$, and therefore the largest allowed time step is given by

$$
\Delta t=\min \left[1.67\left(\frac{h}{c}\right)_{\min }, \frac{1.35}{\sqrt{l_{\max }\left(l_{\max }+1\right)}} \frac{r_{\mathrm{in}}}{c_{\max }}\right],
$$

where $h$ and $c$ are functions of radius and $c_{\max }=c\left(r_{\text {in }}\right)$ is the maximum sound speed in the domain.

\subsection{Lower Boundary Issues}

Pushing the lower boundary deep (like $0.2 R_{\odot}$ ) is certain to ensure a tight CFL restriction, especially if there is a horizontal background structure (like a density inhomogeneity or a flow) that interacts with the acoustics. Consider equations (18) and (19): as one proceeds deeper, the inner radius, $r_{\text {in }}$, reduces and sound speed (see Fig. 1) increases. The time step restriction becomes tighter and consequently the computation becomes very expensive. We have therefore actively made the choice of a spherically symmetric background model with no flows at this depth. Consider also the fact that a large number of waves have already undergone total internal refraction. For example, at a frequency of $5000 \mu \mathrm{Hz}$, the highest $l$ that penetrates a depth of $0.2 R_{\odot}$ is 10 , which means that when determining the CFL condition from equation (20), one need not use the entire spherical bandwidth. In other words, one may replace $l_{\max }$ by $l_{\max }(r)$, denoting the highest $l$ that can propagate at a given radius (given by eq. [9]), a number which deep in the Sun is much smaller than the bandwidth. The resulting time step increase one can obtain by applying this property can be as large as a factor of 2 depending on $l_{\max }$ and the number of radial gridpoints.

\subsection{Buffer Layer}

It was mentioned in $\S 3$ that the transmitting boundary conditions employed in this calculation reflect a large percentage of waves that impact it at significant angles (as opposed to purely radially propagating waves). The effect poses a serious threat to both the stability and the accuracy of the simulation because of this aphysical reflection of waves. To deal with this problem, we insert a buffer layer adjoining the upper boundary in which upward traveling waves are significantly damped out prior to reaching the upper boundary. This ensures that even if these waves are reflected at the boundary, they will have to propagate through the buffer layer again to reach the computational region of interest. This layer serves to diminish the amplitudes of these aphysical waves to insignificance. Thus, in order to prevent un-

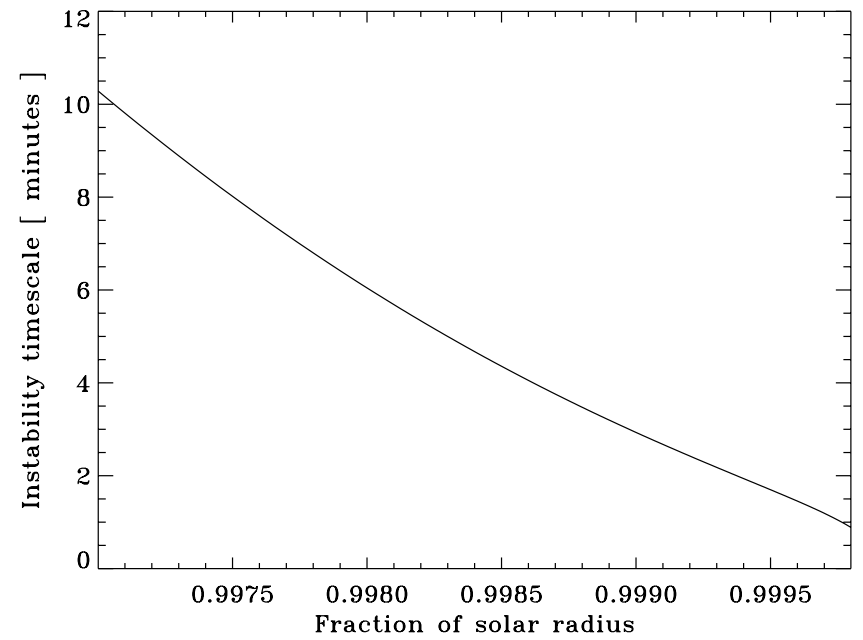

FIG. 4.-Convective instability timescales, $(1 /|N|$ in minutes) as a function of the non-dimensional radius. It can be seen that growth rates of the convective instabilities lie in the same range as the timescales associated with the acoustic waves. The instability arises as a direct consequence of the superadiabaticity of the background model, and since we are not modeling the nonlinear physics of convection, it is crucial that we prevent this linear instability (described in $\S 5.5$ ) from affecting the acoustic signal.

wanted reflections, we introduce buffer layers at each end of the computational domain via the damping term $\Gamma(r) v$ in equation (2).

One problem associated with using such a lower boundary buffer layer is in dealing with waves that have inner turning points located in the midst of the buffer zone. These waves essentially sample the buffer zone and undergo a total internal reflection only to reemerge corrupted by this aphysical layer. The task then is to identify and filter out these waves. As explained in $\S 4$ and specifically in equation (9), we can identify the frequencywavenumber range of waves which propagate to this depth and nullify the corresponding part of the $k-\omega$ spectrum.

\subsection{Convective Instabilities}

The Brunt-Väisälä frequency indicates whether a medium is unstable to convection. It is given by (e.g., Christensen-Dalsgaard 2003, chap. 3)

$$
N^{2}=g\left(\frac{1}{\Gamma_{1}} \frac{\partial \ln p}{\partial r}-\frac{\partial \ln \rho}{\partial r}\right),
$$

where $g$ is gravity, $N$ is the Brunt-Väisälä frequency and $\Gamma_{1}$ is the first adiabatic exponent, defined in equation (4). The solar convection zone extends all the way from roughly $0.7 R_{\odot}$ to the surface. For purposes of discussion, we divide the convection zone into two regions, $0.7 R_{\odot}<r<0.996 R_{\odot}$, where timescales of convective growth are considerably larger than acoustic timescales (5 minutes), and $0.996 R_{\odot}<r<1.0003 R_{\odot}$, where the convective growth rate and acoustic timescales are about equal. Consider the inner region with slowly growing instabilities first. Since we are dealing with a linear system, it might at first sight seem odd that although we restrict acoustic excitation to the bandwidth $2000-5500 \mu \mathrm{Hz}$, we still see instabilities at much lower frequencies. The reasons for this are the finiteness of the excitation time series, which results in the broadening of the frequency response, and numerical round-off errors, which act as broadband sources.

The outer convective envelope introduces difficulties that must be treated with greater care. As can be seen in Figure 4, the instability timescales very close to the surface coincide with 

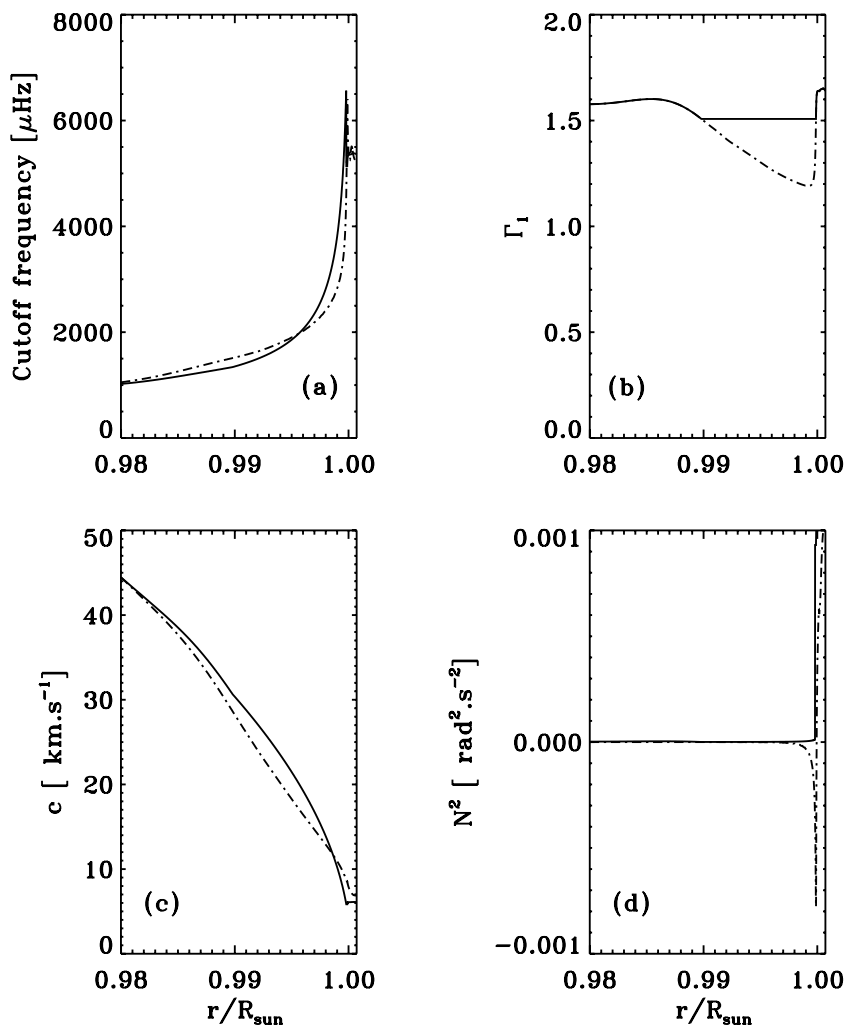

FIG. 5.-Comparison between the properties of the atmosphere given by model S (dot-dashed line) and the artificial model (solid line) used in the computation. The horizontal coordinate of all the above panels is the fractional radius, $r / R_{\odot}$. Panel $(a)$ shows the cutoff frequency dependence with radius; the reflective property of model $\mathrm{S}$ is recovered quite accurately by the artificial model. Panel $(b)$ is a comparison of the first adiabatic exponents; $\Gamma_{1}$ has been altered to render the artificial atmosphere convectively stable. Panel $(c)$ shows sound speed dependence with radius; the layer extending from $0.9998 R_{\odot} \leq r \leq 1.0007 R_{\odot}$ is isothermal, and therefore the sound speed is constant in that region. Panel $(d)$ displays the allimportant measure of convective stability, the Brunt-Väisälä frequency; it can be seen that the artificial model is subadiabatic in the near surface layer, thus ensuring the convective stability of the outer layers.

the center of the acoustic bandwidth. Since our interest lies in capturing the interaction of the acoustics with the background dynamics and not in the direct computation of the convection, we must devise a means to remove this instability without affecting the acoustics. One way to accomplish this is to alter the BruntVäisälä frequencies. A crucial requirement is that the acoustic impedance of the surface layers not be changed by much, since all the acoustic reflection occurs in and around these layers.

It is difficult to alter the timescales of convective growth in the near-surface layers of model S without rendering the background model inconsistent. We have therefore replaced the nearsurface layers given by model $\mathrm{S}$, more specifically the region above $0.98 R_{\odot}$, with an alternative empirical description that satisfies requirements of hydrostatic consistency and convective stability, and preserves the crucial reflective property of the solar atmosphere. Equally essential is that the atmosphere be a smooth extension to the interior (given by model S). Some properties of the empirical model are shown in Figures 5 and 6 and listed in Appendix; more details may be found in Hanasoge (2006). It should be noted that this modification of the background model allows the temporal window of the simulation to be extended to several days; longer simulations, on the order of weeks, are still susceptible to the marginal superadiabaticity exhibited by the interior.
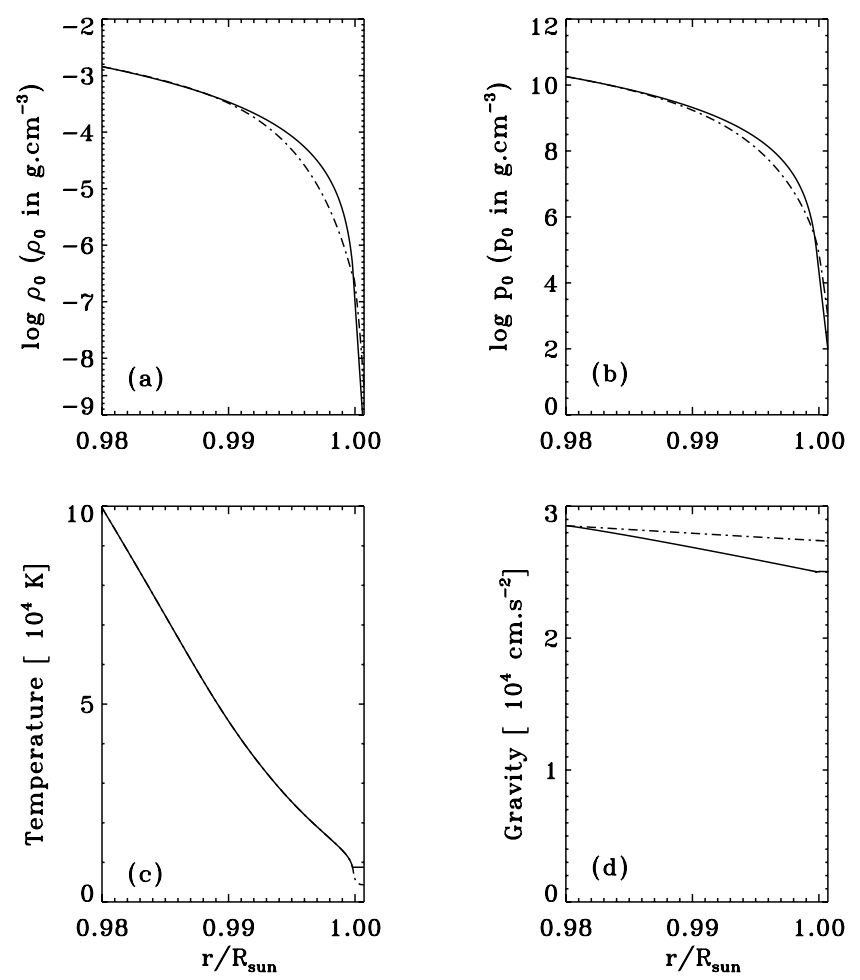

FIG. 6.-Comparison between the properties of the atmosphere given by model S (dot-dashed line) and the artificial model (solid line) used in the computation. The horizontal coordinate of all the above panels is the fractional radius, $r / R_{\odot}$. Panels $(a)$ and $(b)$ show the variation of the logarithmic density and pressure with radius; it can be seen that the two models are quite similar. Panel $(c)$ is a comparison between temperature profiles; the isothermal nature of the outermost layers of the artificial model is visible. Panel $(d)$ shows gravity dependence with radius; the gravity profile of the artificial model is seen to decay more rapidly than that of model S.

\section{VALIDATION}

An important indicator of solar wave structure (acoustic, surface gravity) is the power spectrum, which embodies the frequencywavenumber response of the system to a specific excitation. It is typically shown as the squared Doppler velocity as a function of $\omega$ and $l$. In other words, it is the frequency-wavenumber response of the system to a specific excitation. It can also be understood as a depiction of the resonant modes of the model. A mixture of surface-gravity and acoustic modes appear when solar surface (Doppler) velocities are analyzed. As stated before, the acoustic modes possess maximum power in the frequency range of 2000 $5500 \mu \mathrm{Hz}$. In terms of spatial wavenumbers, the solar power spectrum stretches to extremely high harmonic orders (several thousand), which are at present computationally infeasible. The immediate aim is to replicate some part of the low- to medium$l(0<l<400)$ acoustic spectrum of the Sun in the frequency range described above. We achieve this by exciting waves only in the specified frequency band. Figure 7 is the log power spectrum obtained from a $24 \mathrm{hr}$ long simulation with transmitting lower and upper boundaries placed at 0.226 and $1.0002 R_{\odot}$, respectively. Note that because the short-timescale convective instabilities present in the atmospheric region of the background model have been removed, we see no power at frequencies below $2000 \mu \mathrm{Hz}$.

\section{1. $A D I P A C K$}

A convincing validation of the model is an independent theoretical computation of the resonant modes followed by a comparison 


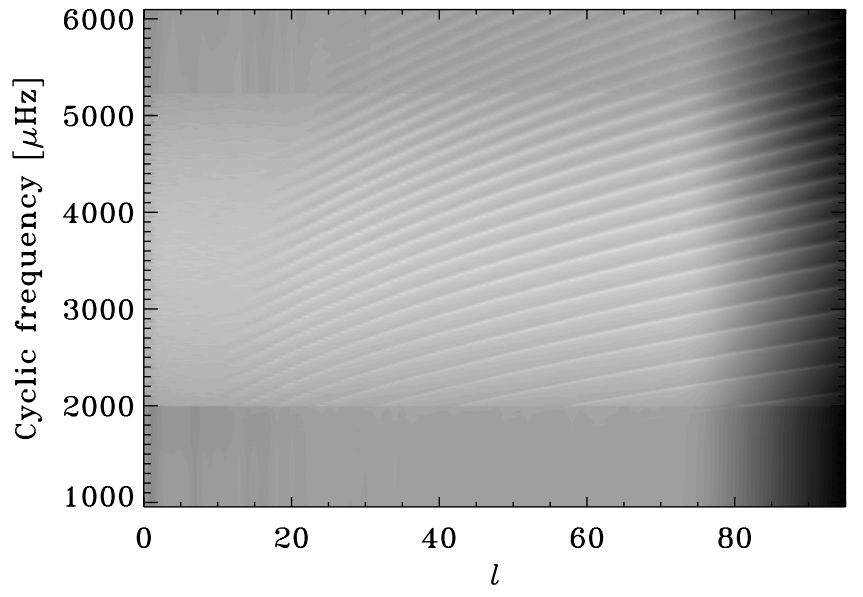

FIG. 7.-Logarithmic power spectrum for a model that extends from 0.226 to $1.00033 R$. The excitation spectrum is a band that approximately encompasses $2000-5500 \mu \mathrm{Hz}$ in frequency and $0-80$ in $l$. The highest $l$ 's contain little or no power to avoid spatial aliasing. Modes with inner turning points deeper than the lower simulation boundary are absent from this spectrum. Note that because the short timescale convective instabilities have been removed, as described in $\S 5.5$, we see no power at frequencies below $2000 \mu \mathrm{Hz}$. This spectrum was extracted from a $24 \mathrm{hr}$ long simulation. [See the electronic edition of the Journal for a color version of this figure.]

with the simulation. ADIPACK (Christensen-Dalsgaard \& Berthomieu 1991) is a software package that may be employed to produce resonant mode data for the spherical shell under investigation. The simulation was performed in a shell that extended from 0.2 to $0.975 R_{\odot}$, with a transmitting lower boundary and a radially oscillating upper boundary. The eigenfrequencies for this simulation were extracted and compared with results from ADIPACK for a similar model. The comparison result in Figure 8 displays good agreement between the ADIPACK modes and the simulation.

\subsection{Shifts in Frequencies due to Rotation}

We now discuss the validation of the model in the presence of a background rotation profile. To ensure that these frequency

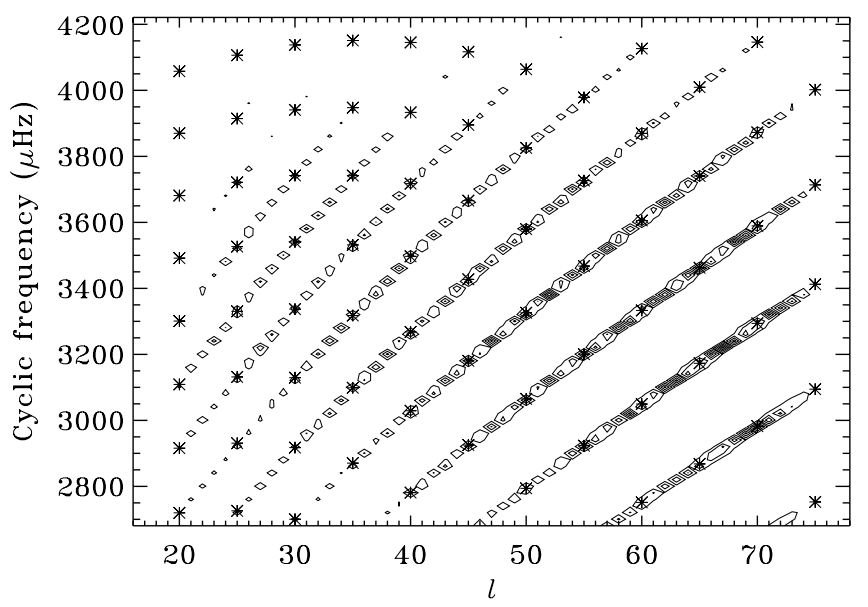

FIG. 8.-Comparison between resonant wavemodes computed by ADIPACK and the simulation. The modes extracted from the simulation are depicted as contours of power, and the ADIPACK frequencies for this model are shown as stars. Note that for ease of comparison, only one in every five modes that are predicted by ADIPACK are depicted. The mode frequencies, located at contour centers, are seen to match closely the frequencies given by ADIPACK. The ridge shapes of the power spectrum extracted from the simulation agree well with those predicted by ADIPACK.

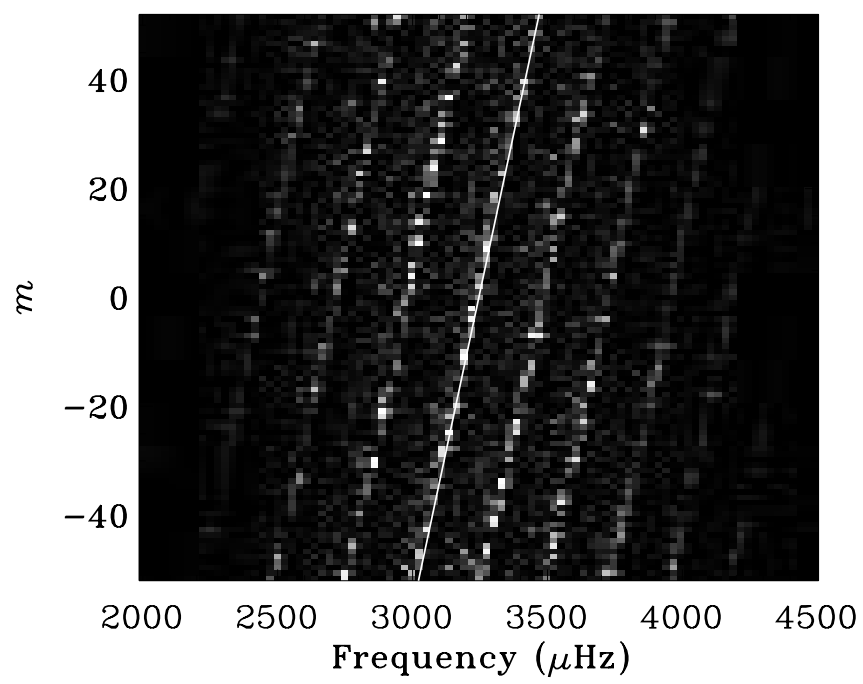

FIG. 9.-The $m-\nu$ power spectrum with $l=52$ for a simulation with pure rotation, the rate being $\Omega / 2 \pi=4300 \mathrm{nHz}$. The solid line shows the analytically calculated trend in the frequency shifts. The frequency resolution of this simulation was $28 \mu \mathrm{Hz}$. In the absence of background flows, contours of maximum power would be lines parallel to the $y$-axis. Rotation causes frequency splitting, shifting prograde modes by $+m \Omega$ and retrograde modes by $-m \Omega$, as indicated by the solid line. Note that as expected, all the (shifted) lines are parallel.

shifts can be observed in a short simulation, we artificially amplify the average solar rotation rate by a factor of 10 ,

$$
\frac{\Omega}{2 \pi}(r, \theta, \phi)=4300 \mathrm{nHz}
$$

In the absence of background flows, resonant wavemode frequencies are characterized only by $l$, having no dependence on $m$. In general, flows induce changes in the resonant frequencies, and specifically, rotation splits the mode frequencies by $\pm m \Omega$, where $m$ is the azimuthal order, depending on whether the modes propagate prograde or retrograde with respect to the direction of rotation. We display the frequency shifts for the $l=52$ set of modes in Figure 9. The frequency resolution of this simulation was $28 \mu \mathrm{Hz}$. The frequency shifts extracted from the simulation match the predicted shifts to within the allowed frequency resolution.

\section{SUMMARY AND CONCLUSIONS}

A method to perform differential studies of the effects offlows and asphericites on the acoustic wave field in full spherical geometry has been proposed. We have designed a technique to attempt the forward problem of helioseismology by performing linear acoustic simulations in an appropriately perturbed solar-like spherical shell. The utility of developing a means to independently test the ability of a helioseismological technique to probe various interior phenomena cannot be understated. Artificial data can also be used to develop the existing methods of helioseismology.

In the past, there have been tests of helioseismology (see, e.g., Jensen et al. 2003) that have involved computations of acoustic wave fields, but none have been performed in spherical geometry. We are currently interested in validating and investigating the inference of meridional flow, farside helioseismology, performing studies of the tachocline, studying signatures of the convective interior, etc.

We have discussed some of the issues associated with a computation of this kind, methods to overcome them, and various techniques used in the validation process. The presence of a 
varying background medium introduces several complications that affect the stability and accuracy of the calculation. For example, the choice of an appropriate radial grid is important to the accuracy of the calculations, given a motivation to minimize computational cost. Wave propagation in such a medium is quite different from propagation in media that traditional aero-acoustic computations are accustomed to. Of course, the side effects of a changing base state can ease and hinder the computation, as has been noted.

We have demonstrated a way of filtering out waves based on their inner turning points, a technique for avoiding tight CFL re- strictions and circumventing instabilities created by an unstable background model. Important to a calculation of this kind is the need to validate the results. We have shown the utility of the process of extracting resonant modes of the domain and techniques to calculate the modes theoretically. The theoretical calculations confirmed the results of the numerical computation.

The authors wish to thank Sebastien Couvidat and Phil Scherrer for their useful comments and suggestions. The work was made possible with funding from grant NASA MDI NNG05GH14G.

\section{APPENDIX}

\section{EMPIRICAL MODEL OF THE NEAR-SURFACE LAYERS}

Properties for the region $0.98 R_{\odot} \leq r \leq 0.9998 R_{\odot},\left(R_{\odot}=6.959894677 \times 10^{10} \mathrm{~cm}\right.$ and $r$ is in centimeters $)$ are given by

$$
\begin{aligned}
p_{0} & =1.8068515 \times 10^{10}\left(\frac{a-r}{0.998989 R_{\odot}-r}\right)^{3.0358} \text { dynes } \mathrm{cm}^{-2}, \\
\rho_{0} & =0.0014415590\left(\frac{a-r}{0.998989 R_{\odot}-r}\right)^{2.0358} \mathrm{~g} \mathrm{~cm}^{-3}, \\
a & =0.998989 R_{\odot}+19.1257\left(r-0.98 R_{\odot}\right)^{2.1} \mathrm{~cm}, \\
g & =-\frac{1}{\rho_{0}} \frac{d p_{0}}{d r}, \\
T_{0} & =T_{0 S}, \\
\Gamma_{1} & =\max \left(\Gamma_{1 S}, 1.4921\right),
\end{aligned}
$$

where the subscript $S$ refers to properties described by model S. The region $0.9998 R_{\odot} \leq r \leq 1.0007 R_{\odot}$ is described by

$$
\begin{aligned}
p_{0} & =22319.813 \exp \left(\frac{R_{\odot}-r}{H}\right) \text { dynes } \mathrm{cm}^{-2}, \\
\rho_{0} & =9.8535179 \times 10^{-8} \exp \left(\frac{R_{\odot}-r}{H}\right) \mathrm{g} \mathrm{cm}^{-3}, \\
H & =9 \times 10^{6} \mathrm{~cm}, \\
g & =25033.357 \mathrm{~cm} \mathrm{~s}^{-2}, \\
T_{0} & =8727 \mathrm{~K}, \\
\Gamma_{1} & =\max \left(\Gamma_{1 S}, 1.4921\right) .
\end{aligned}
$$

\section{REFERENCES}

Belousov, S. L. 1962, Tables of Normalized Associated Legendre Polynomials (New York: Macmillan)

Christensen-Dalsgaard, J. 2003, Lecture Notes on Stellar Oscillations, (5th ed.; Aarhus: Aarhus Univ.), http://astro.phys.au.dk/ jcd/oscilnotes/

Christensen-Dalsgaard, J., \& Berthomieu, G. 1991, in Solar Interior and Atmosphere, ed. A. N. Cox, W. C. Livingston, \& M. Matthews (Tucson: Univ. Arizona Press), 401

Christensen-Dalsgaard, J., et al. 1996, Science, 272, 1286

Colonius, T., \& Lele, S. K. 2004, Prog. Aerosp. Sci., 40, 345

Deubner, F.-L., \& Gough, D. O. 1984, ARA\&A, 22, 593

Georgobiani, D., Stein, R. F., \& Nordlund, Å. 2003, ApJ, 596, 698

Hanasoge, S. M. 2006, in ILWS Workshop, The Solar Influence on the Heliosphere and Earth's Environment, (Goa: ILWS), http://ilws.gsfc.nasa.gov/ ilwsgoa_hanasoge.pdf
Hu, F. Q., Hussaini, M. Y., \& Manthey, J. L. 1996, J. Comput. Phys., 124, 177 Jensen, J. M., Olsen, K. B., Duvall, T. L., \& Jacobsen, B. H. 2003, in Proc. of SOHO $12 /$ GONG+2002, ed. H. Sawaya-Lacostep (ESA SP-517; Noordwijk: ESA), 319

Lele, S. K. 1992, J. Comput. Phys., 103, 16

Lui, C. 2003, Ph.D. thesis, Stanford Univ.

Orszag, S. A. 1970, J. Fluid Mech., 41, 363

Rosenthal, C. S., et al. 1999, A\&A, 351, 689

Thompson, K. W. 1990, J. Comput. Phys., 89, 439 